\title{
Replication and Open Science in Applied Linguistics Research
}

Kevin McManus, Penn State University, USA, kmcmanus@psu.edu

\begin{abstract}
This chapter addresses the role and place of replication research and open science practices in advancing theory building and new research directions in the field of applied linguistics. The chapter begins by describing what replication research is, what the most common types of replication study are, and why carrying out replication matters. Close attention is paid throughout to the ways in which replication benefits from and contributes to a variety of open science initiatives, including open materials, open access and preprints, and preregistration.
\end{abstract}

\section{Introduction}

Interest, curiosity, and, to some extent, consensus about the role and place of replication research in the field of applied linguistics is growing (McManus, 2021). For example, some journals now include replication studies as a specific manuscript type (e.g., Applied Psycholinguistics, Language Teaching, Studies in Second Language Acquisition), workshops, summer schools, and book-length guides are helping researchers design and disseminate replication research (e.g., Porte \& McManus, 2019), reviews of the field have synthesized current practices (e.g., Marsden et al., 2018), funding bodies are investing in the replication of influential studies (e.g., Dutch Research Council, Institute of Education Sciences), and professional organizations now include replication studies as highly-valued scholarly products in their guidelines for tenure and promotion (e.g., American Association for Applied Linguistics). At the same time, the field of 
applied linguistics has recently invested in various practices designed to improve the openness, transparency, and reproducibility of research. Some journals now award 'Open Practice Badges' for articles that share data, materials, and/or preregister their analyzes (e.g., Bilingualism: Language and Cognition, Language Learning), funding bodies are beginning to require that project data are made fully available and usable by others (e.g., Economic and Social Research Council, National Science Foundation), and preprint repositories as well as open-access journals are allowing research products to be made publicly available (e.g., Journal of the European Second Language Association, OSF Preprints). Taken together, recent developments in the field point to an increasing awareness about the need and importance of replication and transparency in the growth and strengthening of a discipline (Gass et al., 2021; Marsden, 2019).

As other commentators have noted, these developments have mostly been in response to a so-called 'replicability crisis' (see Al-Hoorie \& Hiver, this volume), first documented in the field of psychology through large-scale attempts to replicate classic and contemporary published findings (Klein et al., 2014; Open Science Collaboration, 2012, 2015). Difficulties replicating previously published findings in psychology led to widespread concern that most published findings may be false due to selective publication, questionable research practices, and a lack of replication (Cumming \& Calin-Jageman, 2017; Ioannidis, 2005). Conducting replication studies and integrating open science (OS) practices into the research process are understood to be some of the ways to address wide-spread concern about the nature and validity of published research.

Even though no large-scale replication effort has been attempted in our field, the reproducibility and replicability of applied linguistics research still represents a talking point. This is because replication is the principal way to move us towards a more nuanced, finergrained understanding about the nature and a validity of a specific study's findings (Gould \& 
Kolb, 1967; Porte \& McManus, 2019; Schmidt, 2009). In applied linguistics, determining the validity and reliability of published findings is critical given the impact of unsuccessful replications in neighboring disciplines (Klein et al., 2014) and the observation that much of our theoretical understanding is built on a small number of influential studies that have not been replicated (e.g., Bailey et al., 1974; for review, see Myles, 2010). Indeed, Marsden et al.'s (2018) review suggested that the amount of replication in our field is worryingly low, despite numerous calls over the years to engage in more replication (see also Porte, 2012; Porte \& Richards, 2012). One interpretation of this situation is that we are not systematically revisiting previous studies to better understand their contribution to knowledge. Because no single study can provide all the answers, our field needs replication, along with meta-analysis and transparent practices, so that we can better understand previous research (Allen \& Preiss, 1993; Plonsky, 2012). This is why replication is vital to the growth and consolidation of a discipline's evidence base: it allows us to understand how a study's research data were collected, measured, and analyzed, as well as the extent to which unexpected and/or unanticipated factors potentially shaped the conclusions.

In a recent survey of the attitudes and practices of second language researchers' toward replication, however, McManus (2021) found that researchers' evaluated replication studies as very relevant and valuable to the field. In addition, claims that replication studies lacked originality and/or innovation were not supported. Contrary to previous reviews of the field (e.g., Porte \& Richards, 2012), therefore, these findings indicated that negative attitudes alone do not appear to explain the lack of replication in our field. Instead, it is possible that what we might be perceive as a lack of replication might reflect a lack of clear labelling of replication studies. This is because half of published replication studies in the sample lacked explicit labeling and one 
quarter of completed replications were reported to be unpublished. These are important issues to address in order to improve the discoverability of replication studies their potential impact on applied linguistics research.

In the field of applied linguistics, replication studies are critical given that one line of this research seeks to better understand how speakers learn and use additional languages. In order to investigate these questions, research has used experimental designs to examine "how the systematic manipulation of the mechanisms of learning and/or the conditions under which they occur enable or facilitate the acquisition of an additional language" (Loewen, 2020, p. 2). The key term here is "systematic manipulation", which is what replication involves, especially close and approximate replications. A close replication revisits a specific study, systematically manipulates a variable of interest, and then repeats the study while aiming to keep all other aspects of the study unchanged. In this way, replication investigates in what ways this intentional variable modification might alter the study's findings. An extension study, in contrast, does not share this aim. In an extension study, the motivation comes from the findings of multiple studies to investigate new research questions, using new methodologies and/or new analyses. The key difference is this: a replication systematically repeats a previous study (with or without changes) to contribute a more nuanced understanding of that study and its findings, while an extension study builds on what we know about a particular phenomenon by taking an existing line of research in new directions, applying it in new contexts, etc. Thus, replication is key to growing and strengthening a discipline precisely because it draws conclusions through systematic manipulation, repetition, and comparison with a previous study. However, for a replication study to be closely comparable to the initial study, the replication must be able to closely follow the design and analyses procedures of the initial study. This is where OS and replication work 
together: Making materials and analysis/coding protocols available to others is critically needed to facilitate replication (for examples of these practices, see Arroyo \& Yilmaz, 2018; Saito et al., 2020).

In this chapter, the aim is to discuss some of the key components of replication research in the field of applied linguistics and the specific ways in which replication both benefits from and contributes to OS. We begin by presenting some common types of replication research in our field, with examples, drawing attention to the ways in which OS practice can contribute to the conduct of high-quality replication studies. This discussion is followed by recommendations for integrating OS practices into the replication research process.

\section{Common options for replication research in applied linguistics}

When we carry out a replication study, our aim is to design, report, and compare a piece of research that repeats a previous study in some way. The extent to which a replication study is different from the initial study must be reported both in the study title and in the text (Appelbaum et al., 2018). Common labels used in replication research to indicate the amount of change with the original study include 'exact replication', 'close replication' (or partial), 'approximate replication', and 'conceptual replication' (or approximate). We will briefly review these main types of replication study before looking in detail at an example of a close replication study. We use this case study approach to illustrate how a replication study is different from an extension study as well as some of the decisions that go into the replication research process.

In an exact replication, the initial study's entire procedure is followed without alteration. This means that the data sample (i.e., participants) is the same, the research questions and design are the same, and the coding and analyses are the same. Clearly, carrying out an exact replication 
is probably one of the most difficult types of replication study to do, if not impossible. This is because even though it might be possible to locate and use the initial study's materials and analyses, it is very likely that we would struggle to keep the data sample the same. Of course, the sample could be similar (e.g., ESL learners in Australia vs. United Kingdom), but a variety of factors will make the population different (e.g., context, time, individual participant backgrounds). This is one reason why exact replication in the social sciences is immensely difficult if not "an unachievable objective" (Porte \& McManus, 2019, p. 72). Indeed, Nosek and Errington (2020, p. 3) claim that "there is no such thing as exact replication", largely because there are always differences between the initial study and the replication. One likely exception to this, however, includes corpus-based work since corpora are time-stamped and the content included in a corpus does not change over time, contexts, etc. As long as the replication has access to the same corpus as used in the initial study, then an exact replication can be possible in our field. Of course, using the same corpus as a previous study does not make it an exact replication unless all other aspects of the study are also repeated without changes.

A close replication is the most achievable type of replication in our field that allows for the most comparison with the initial study. This is because in a close replication study only one major variable is modified. All other aspects of the original study are kept as constant as possible. This means that a close replication study could modify, for example, the instruction, or the outcome measure, or the $\mathrm{L} 2$, or the $\mathrm{L} 1$ in order to understand how modifying that variable influenced the study's outcomes. A close replication is perhaps the clearest way to advance knowledge and understanding in a field because it sets out to investigate how intentionally modifying a single variable impacts the study's findings (see Porte \& McManus, 2019). 
An approximate replication is quite similar to a close replication but with more room for modification. Here, two variables are modified (Porte \& McManus, 2019). Those changes are then compared with initial study, following the same line of comparison as discussed for close replications. This means that all other aspects of the initial study remain unmodified.

The last type of replication to be discussed here is conceptual replication. A conceptual replication allows for almost all methodological aspects of the initial study to be changed and involves "repetition of a test of a hypothesis or a result of earlier research with different methods" (Schmidt, 2009, p. 91). In other words, a conceptual replication asks the same general research question or tests the same hypothesis as the initial study but can draw on a different research methodology (e.g., different sample, different methods, different analyses). In contrast to close and approximate replications that contain a small amount of variation when compared to the initial study, drawing comparisons in a conceptual replication tends to be more difficult because many differences can exist between the studies. This is not to say that conceptual replications are not important, though. A conceptual replication just has a different set of aims. Rather than seeking to verify and consolidate the claims of a previous study by systematically manipulating its methodology, conceptual replications instead work towards establishing claims about theories and concepts. In addition, conceptual replications struggle to better understand the specifics of an individual study because the extent of comparison between studies is necessarily limited. This is an important point: the more that is different between the studies, the more difficult it is to draw comparisons. In this sense, conceptual replications are more like extension studies: A conceptual replication might ask the same question as some previous study, but it investigates this question in a different way. 
In the rest of this section, we look at a recent replication study in applied linguistics as a case study to understand some of the ways in which close replication can be carried out. Our focus is on reviewing this approach to replication to understand (i) the rationale/motivation for close replication, (ii) how replication was approached, and (iii) how the replication benefited from and/or contributed to OS.

Close replication: McManus and Liu (2021)

McManus and Liu (2021) is a close replication of Wu and Ortega (2013). As with any discussion of replication, we need to start with the initial study because this is where the motivation for replication begins. Wu and Ortega (2013) investigated the extent to which a Mandarin Chinese elicited imitation test (EIT) could distinguish between high and low oral language proficiency abilities. Participants were instructed L2 learners and heritage speakers of Mandarin, sampled from (i) second-level university courses to represent low-level language abilities and (ii) third-, fourth-, and graduate-level university courses to present high-level language abilities. In this EIT test, participants listened to a sentence (e.g., 我得去剪头发了 'I have to get a haircut') and repeated the sentence as closely as possible following a short pause. The sentences increased in syllable length and complexity over the course of the test. Performance on the EIT showed that learners in the high group performed more accurately than learners in the low group. In addition, EIT scores correlated positively with performance on an oral narrative task. Taken together, these findings indicated that the EIT was able to distinguish between different levels of oral language abilities. Given these promising findings, EITs for L2 proficiency assessment in other languages have emerged over the years with similar results, adding weight to the usefulness of 
the EIT as a L2 proficiency assessment tool in our field (e.g., Kim et al., 2016 for L2 Korean; Tracy-Ventura et al., 2014 for L2 French; see also Kostromitina \& Plonsky, 2021).

McManus and Liu's (2021) close replication set out to better understand $\mathrm{Wu}$ and Ortega's (2013) findings, partly motivated by the observation that interest in the use of EIT to measure L2 proficiency is growing. Of particular interest was how the initial study sampled participants according to language abilities. The initial study's participants were recruited from lower-division courses (200-level courses) and upper-division courses (300-level, 400-level, and graduate-level courses). Those in the lower-division courses were assigned to the low group and those in the upper-division courses were assigned to the high group. Compared to the low group, therefore, language ability and classroom learning experience in the high group was more varied because these learners were sampled from three curricular levels. To address this potential influence on the findings (i.e., greater curricular and ability variation in the high group), the close replication narrowed sampling in the high group to include students enrolled in 300- and 400level courses only. This sampling modification allowed us to understand the extent to which including graduate students in the high group, arguably the learners with the most advanced language abilities, contributed to W\&O's findings. In addition, we recruited participants from 100-level courses to create a beginner group (not part of W\&O's design). Our rationale for these changes was to understand: (1) the ways in which the broad grouping of language abilities in the high group contributed to W\&O's findings and (2) the extent to which W\&O's EIT can additionally distinguish between finer-grained language abilities (by comparing beginner, low, and high language abilities). Therefore, the purpose of McManus and Liu's (2021) close replication study was to better understand how participant sampling contributed to conclusions about the EIT's effectiveness for assessing L2 oral language abilities. 
The close replication study used the same data collection materials (available from IRIS), recruited L2 learners that matched the criteria of initial study, followed the same data collection procedures, and analyzed the data using the same coding and analysis protocols as described in the initial study. Thus, by keeping all other aspects of the initial study the same, the replication study served to better understand how participant sampling and group membership contributed to EIT performance as reported in the initial study.

Overall, the replication's results patterned very similarly to those in the initial study. No reliable differences were found between the studies for mean EIT performance in the low and high groups (assessed using Cohen's $d$ effect sizes and 95\% confidence intervals for $d$, see McManus \& Marsden, 2018; Porte \& McManus, 2019). Within-group comparisons in the replication showed that performance was more accurate as a function of language ability grouping, consistent with Wu and Ortega. That is, mean EIT scores were highest in the high group, followed by the low group, and were lowest in the beginner group. Effect size comparisons showed that the magnitudes of these between-group differences were medium to large. In terms of performance on the oral narrative, similar patterns of results were found: medium to large differences between the replication's different language ability groups.

Taken together, the close replication demonstrated that performance in the EIT mirrored performance in the oral narrative by distinguishing between the different language ability groups. Correlations between the EIT and the oral narrative task indicated a high degree of shared variance. Thus, the replication provided strong evidence in support of the initial study's claim that the Mandarin EIT can function as an effective L2 proficiency measurement tool. In addition, this replication's findings for beginning learners indicated that the EIT can be used with inexperienced and low proficiency L2 learners. This is an important contribution to our 
understanding of the EIT since most previous research has assessed EITs among intermediate-toadvanced L2 speakers.

In terms of OS practices, the initial study's testing materials were made available to others via the IRIS digital repository, a free and public resource that allows researchers to upload and download "instruments, materials, stimuli, and data coding and analysis tools used for research into second languages" (IRIS, 2021; see also Marsden et al., 2016). Not only does this OS practice facilitate replication of the initial study, but it also permits further replication. For example, McManus and Liu's replication modified the data sample, but future work could additionally modify features of the EIT, learning context, and/or coding and analysis as a way to systematically explore the role of each of these variables. In addition, the initial study made the coding and scoring protocols publicly available so that other research teams can implement the same coding and scoring decisions as the initial study. These are important steps that facilitate replication and subsequent comparison.

In sum, this close replication study set out to better understand to what extent sampling decisions contributed to L2 performance on the Mandarin EIT as reported in Wu and Ortega (2013). It achieved that goal by modifying recruitment to the high group and adding a new group of beginner learners. In this way, the close replication study was able to better understand (i) how the broader sampling of language abilities in the high group contributed to the study's findings and (ii) whether the EIT was able to additionally distinguish between finer-grained language abilities (e.g., beginner-low).

\section{Integrating Open Science practices into Replication}


Up until this point, we have considered different options for replication studies in the field of applied linguistics, including a case study of a close replication. In this section, recommendations for integrating OS practices as means to both facilitate and extend replication research are discussed. We focus on three practices: open materials (section 3.1), open access publishing and preprints (section 3.2), and preregistration (section 3.3). These three OS practices are prioritized because they help address some of the most common obstacles to carrying out and reporting replication studies in our field (see also Marsden et al., 2018; Porte \& McManus, 2019).

\subsection{Open materials}

In the opening section of this chapter, we mentioned that some journals in our field have recently awarded 'Open Practice Badges' to articles that have shared materials, data, and/or preregistered their analyses prior to data collection. In order for a paper to receive a badge for open materials, the journal Studies in Second Language Acquisition (SSLA), for example, notes the following:

This badge is awarded to researchers who share their materials publicly so they can be used to replicate research procedures and analyses. These materials should be shared on an open-access repository such as the IRIS Digital Repository of Data Collection Materials (http://www.iris-database.org) and be accompanied by an explanation of how the materials relate to a given methodology.

(SSLA, 2021)

For an article to receive an open materials badge in SSLA, therefore, that article must have made their data collection tools available on an open-access repository and those materials must include some type of description and explanation of how those materials were used in the published study. To do this, researchers can use platforms such as IRIS, OSF, an open-access university repository, or an independent repository at www.re3data.org. 
In the previous quote, SSLA state that one aim of sharing materials is so a published study's procedures and analyses can be replicated. This is clearly an important reason for sharing materials. It is also important to note that making the data collection tools available is a very helpful way to increase the transparency of the research. In particular, it helps readers more fully understand what went on in that study, how the research data were collected, what tools were used, and what those tools looked like. In short, providing the materials used can provide a fuller account than the descriptions provided in the published paper.

It is also important to note that making a study's materials available facilitates the conduct of high-quality replication studies. When a study does not make these available, it makes the replication process more difficult, if not impossible. This is because the research team must attempt to recreate the materials from the description and/or contact the authors for the materials. These paths can lead to mixed success depending on the age of the initial study and the clarity of the study's methodological reporting.

When it comes to replication, Marsden et al.'s (2018) review of 67 self-identified replication studies indicated that few of the initial studies had made their materials fully accessible. Of the 67 self-identified replications, only three initial studies had made their full data collection materials available. However, all were located behind a paywall, meaning that the accessibility of these materials was restricted. Indeed, as Marsden et al. (2018) mention, this is a worrying state of affairs since replication of these studies would have involved locating the materials using other means and/or recreating the materials. Taking these steps clearly has a serious impact on the quality of the replication. We cannot be confident in the interpretation of a replication study if the materials are different. As discussed in Porte and McManus (2019), 
replications should report how the initial study's materials were accessed and/or whether these materials were recreated in part or in whole.

\subsection{Open access and preprints}

A critical component for the advancement of applied linguistics research is that research findings need to be discoverable, accessible, and useable. What this means, therefore, is that research products need to be made available to other users. We are beginning to see changes to how scholarly work is made available to other users, including the development of open access publishing models as well as OS practices such as open materials and open data. Some social science disciplines are additionally using preprints to quickly disseminate and make research publicly available. Before looking at preprints and the ways in which they can promote the availability of our research products while also facilitating replication, we will take a moment to review open access publishing in applied linguistics.

An open access journal publishes content that is fully and freely accessible to others. This typically means that an article can be downloaded from the journal's publishing platform at no cost to the reader. This contrasts with non-open access journals that publish articles behind a paywall, meaning that users require some type of privileged access (e.g., via a university library that subscribes to that journal) or must pay to access the research product. There are now instances of hybrid journals in our field (e.g., Annual Review of Applied Linguistics), in which non-open access journals will allow authors to make their paper open access by paying some type of processing cost. At the same time, our field has recently seen a number of initiatives to launch open-access journals, which are journals that are fully accessible to users and do not require authors to pay processing costs).(e.g., Canadian Journal of Applied Linguistics, Studies 
in Second Language Learning and Teaching, TESL-EJ,; for a list of open access journals in applied linguistics, see Al-Hoorie, 2021) Taken together, open access means that an article is free and fully accessible to readers (i.e., it is not restricted to privileges users).

An additional initiative consistent with OS is the creation and development of preprint platforms such as OSF Preprints and PsyArXiv. Although this practice does not yet appear to be as common in applied linguistics as in other social science disciplines (e.g., psychology), a preprint refers to a "complete written description of a body of scientific work that has yet to published in a journal" that is made available to users on some type of freely accessible platform such as OSF Preprints (Bourne et al., 2017, p. 1). A preprint can include a paper that is ready to be submitted to a journal for peer-review as well as a paper that has already been peer-reviewed and is awaiting final processing by a journal. In short, a preprint paper is a fully accessible report of scholarly work that authors make publicly accessible to other users. Before the development of preprint servers, some researchers would make preprint versions of their work available on personal websites. The difference with the creation of preprints is that these are housed on accessible platforms that are associated with a digital object identified (or DOI) so that the preprint can be cited.

While an important aspect of preprints is that they can speed up dissemination and discoverability of scholarly work, they also serve an important function in facilitating access to scholarly work. At a time when we are increasingly asking questions about how to make research available and accessible, the use of preprints is an important advancement towards this goal. Preprints also make an important contribution towards replication precisely because they improve access to scholarly work. This is important given that some reviews of the field suggest that replications can be more difficult to publish because they are perceived to lack excitement 
and novelty (see Porte \& Richards, 2012), meaning that publication bias can prevent the timely dissemination and accessibility of important replication studies. Preprints work to address this problem by making the research discoverable and usable by others, irrespective of the findings and perceived novelty and /or perceived excitement of the work.

\subsection{Preregistration}

Research planning, or preregistration, is the last of the OS practices discussed in this chapter. Planning is an aspect of the research process that we are all familiar with, which makes preregistration quite a familiar concept in many regards. For example, as graduate students we design and plan out a $\mathrm{PhD}$ project before collecting and analyzing data. In many educational systems, this involves a $\mathrm{PhD}$ proposal defense, where members of a $\mathrm{PhD}$ committee review and critique the proposed study and analysis. This proposal is then approved before data collection begins. We also encounter research planning when we seek to obtain ethics or IRB approval. Typically, this process requires us to think through how the data will be collected, how they will be analyzed, and a series of other methodological, analytical, and ethical consideration. In addition, when we apply for a grant, we also have to plan and justify a research plan, including decisions about data collection, coding and analysis. These are just some of the ways in which we plan out research projects before we collect and analyze our data. Taken together the concept of preregistration is familiar to us in the sense that it is about planning before execution.

As discussed in other chapters in this volume (e.g., Huensch, this volume), preregistration is thought to be one way to reduce the risk of questionable research practices (e.g., making decisions about analysis and coding after seeing the data, see Cumming \& Calin-Jageman, 2017; John et al., 2012). It achieves this goal by encouraging scholars to state their research plans in 
advance of collecting data, then registering these research plans on a platform like $O S F$ Registries (https://osf.io/prereg/). The registration is then saved. The option of a 'public registration' means that the research plan becomes a frozen, non-editable version of the proposed project. As a result, the method and analyses that the researchers registered are those that should be carried out (but preregistration does not prevent additional analyses from being carried out too, of course, see Huensch, this volume). This type of planning followed by the creation of a public version of that planning is thought to be one way to reduce the risk of questionable research practices. In addition to this form of preregistration, some journals in our field have launched Registered Reports as a specific manuscript type (e.g., Bilingualism: Language and Cognition, Language Language). A Registered Report is different in that the registration is peerreviewed by the journal before data collection, leading to an in-principle accept when successful (see Huensch, this volume; Hui \& Huntley, this volume).

Even though preregistration might sound preventative in the sense that one of its aims is to try to reduce (or prevent) scientific misconduct (see Yamada, 2018), preregistration has a remarkably helpful feature in that it encourages us to think through our study designs before data collection. This is particularly helpful for the conduct of close and approximate replication studies because these approaches to replication involve repeating a study with a small amount of intentional variation. A useful tool to facilitate this process is the preregistration template for replication studies (Brandt et al., 2014). This template includes a list of primer questions to help researchers plan their replications before collecting data. We will take two of the questions from Brandt et al.'s Replication Recipe template to illustrate how using a planning template can help facilitate the process of variable selection and modification in a replication study. 
One question from the template is as follows: "What is the effect you are trying to replicate?" Here, the aim is to think through what the aim of the replication study is (i.e., why are you going to conduct a replication study?). In the McManus and Liu (2020) example, a close replication was carried out to verify the claim that the Chinese EIT was able to reliably distinguish between different two instructional levels. In a close or approximate replication, the aim is to replicate the same effect as reported in the initial study. To achieve this goal, you are most likely going to be modify some independent variable.

A second question from the template that is important to consider is as follows: "What differences between the original study and your study might be expected to influence the size and/or direction of the effect?" This question encourages you to think about how the identified variable modification might change the effect you are trying to replicate. In short, you are making a prediction based on what you know about the field. In McManus and Marsden (2018), for example, the variable modification was the type of instruction. This was modified to understand how changes to the initial study's instruction contributed to L2 learning outcomes. The replication's predictions thus required an account for why changing the instruction might influence the size and/or direction of the effect. To do this, the replication drew on previous research about the role of explicit information in L2 learning (e.g., Marsden \& Chen, 2011; Sanz \& Morgan-Short, 2004).

In summary, planning is an integral component of the research process that applies to replication studies just as much as it does to other types of research. Before carrying out a replication study, it is important to plan what variable(s) will be modified and why. Brandt et al.'s (2014) Replication Recipe template is a helpful tool for replication planning. 


\section{Conclusion}

In this chapter, we have discussed the role and place of replication research and OS practices in advancing theory building and new research directions in the field of applied linguistics. We started with a review of what replication research is, what the most common types of replication study are, and why carrying out replication matters. We discussed some of the ways that replication benefits from and contributes to a variety of OS practices, including open materials, open access and preprints, and preregistration. Recommendations for integrating OS practices into the replication research process were also provided.

\section{References}

Al-Hoorie, A. H. (2021, October 27). List of open access journals. Ali H. Al-Hoorie. https://www.ali-alhoorie.com/applied-linguistics-open-access-journals

Allen, M., \& Preiss, R. (1993). Replication and meta-analysis: A necessary connection. Journal of Social Behavior and Personality, 8(6), 9-20.

Arroyo, D. C., \& Yilmaz, Y. (2018). An Open for Replication Study: The Role of Feedback Timing in Synchronous Computer-Mediated Communication: Role of Feedback Timing. Language Learning, 68(4), 942-972. https://doi.org/10.1111/lang.12300

Bailey, N., Madden, C., \& Krashen, S. D. (1974). Is there a "natural sequence" in adult second language learning? Language Learning, 24(2), 235-243. https://doi.org/10.1111/j.14671770.1974.tb00505.x

Bourne, P. E., Polka, J. K., Vale, R. D., \& Kiley, R. (2017). Ten simple rules to consider regarding preprint submission. PLOS Computational Biology, 13(5), e1005473. https://doi.org/10.1371/journal.pcbi.1005473 
Brandt, M. J., Ijzerman, H., Dijksterhuis, A., Farach, F. J., Geller, J., Giner-Sorolla, R., Grange, J. A., Perugini, M., Spies, J. R., \& van ’t Veer, A. (2014). The Replication Recipe: What makes for a convincing replication? Journal of Experimental Social Psychology, 50, 217224. https://doi.org/10.1016/j.jesp.2013.10.005

Cumming, G., \& Calin-Jageman, R. (2017). Introduction to the new statistics: Estimation, open science, and beyond. Routledge.

Gass, S. M., Loewen, S., \& Plonsky, L. (2021). Coming of age: The past, present, and future of quantitative SLA research. Language Teaching, 54(2), 245-258. https://doi.org/10.1017/S0261444819000430

Gould, J., \& Kolb, W. L. (Eds.). (1967). A Dictionary of the Social Sciences. Tavistock Publications.

Ioannidis, J. P. A. (2005). Why Most Published Research Findings Are False. PLoS Medicine, 2(8), e124. https://doi.org/10.1371/journal.pmed.0020124

IRIS. (2021, October 27). IRIS. A digital repository of instruments and materials for research into second languages. https://www.iris-database.org

John, L. K., Loewenstein, G., \& Prelec, D. (2012). Measuring the Prevalence of Questionable Research Practices With Incentives for Truth Telling. Psychological Science, 23(5), 524532. https://doi.org/10.1177/0956797611430953

Kim, Y., Tracy-Ventura, N., \& Jung, Y. (2016). A Measure of Proficiency or Short-Term Memory? Validation of an Elicited Imitation Test for SLA Research. The Modern Language Journal, 100(3), 655-673. https://doi.org/10.1111/modl.12346

Klein, R. A., Ratliff, K. A., Vianello, M., Adams, R. B., Bahník, Š., Bernstein, M. J., Bocian, K., Brandt, M. J., Brooks, B., Brumbaugh, C. C., Cemalcilar, Z., Chandler, J., Cheong, W., 
Davis, W. E., Devos, T., Eisner, M., Frankowska, N., Furrow, D., Galliani, E. M., ... Nosek, B. A. (2014). Investigating Variation in Replicability: A "Many Labs"

Replication Project. Social Psychology, 45(3), 142-152. https://doi.org/10.1027/18649335/a000178

Kostromitina, M., \& Plonsky, L. (2021). Elicited imitation tasks as a measure of L2 proficiency: A meta-analysis. Studies in Second Language Acquisition, 1-26. https://doi.org/10.1017/S0272263121000395

Loewen, S. (2020). Introduction to instructed second language acquisition. Routledge.

Marsden, E. (2019). Open science and transparency in Applied Linguistics research. In The Encyclopedia of Applied Linguistics (pp. 1-10).

Marsden, E., \& Chen, H.-Y. (2011). The Roles of Structured Input Activities in Processing Instruction and the Kinds of Knowledge They Promote: Roles of Structured Input Activities in Processing Instruction. Language Learning, 61(4), 1058-1098. https://doi.org/10.1111/j.1467-9922.2011.00661.x

Marsden, E., Mackey, A., \& Plonsky, L. (2016). The IRIS Repository: Advancing research practice and methodology. In A. Mackey \& E. Marsden (Eds.), Advancing methodology and practice: The IRIS Repository of Instruments for Research into Second Languages (pp. 1-21). Routledge.

Marsden, E., Morgan-Short, K., Thompson, S., \& Abugaber, D. (2018). Replication in second language research: Narrative and systematic reviews and recommendations for the field. Language Learning, 68(2), 321-391. https://doi.org/10.1111/lang.12286 
McManus, K. (2021). Are replication studies infrequent because of negative attitudes? Insights from a survey of attitudes and practices in second language research. Studies in Second Language Acquisition.

McManus, K., \& Liu, Y. (2021). Using elicited imitation to measure global oral proficiency in SLA research: A close replication study. Language Teaching, 1-20. https://doi.org/10.1017/S026144482000021X

McManus, K., \& Marsden, E. (2018). Online and offline effects of L1 practice in L2 grammar learning: A partial replication. Studies in Second Language Acquisition, 40(2), 459-475. https://doi.org/10.1017/S0272263117000171

Myles, F. (2010). The development of theories of second language acquisition. Language Teaching, 43(3), 320-332. https://doi.org/10.1017/S0261444810000078

Nosek, B. A., \& Errington, T. M. (2020). What is replication? PLOS Biology, 18(3), e3000691. https://doi.org/10.1371/journal.pbio.3000691

Open Science Collaboration. (2012). An Open, Large-Scale, Collaborative Effort to Estimate the Reproducibility of Psychological Science. Perspectives on Psychological Science, 7(6), 657-660. https://doi.org/10.1177/1745691612462588

Open Science Collaboration. (2015). Estimating the reproducibility of psychological science. Science, 349(6251), aac4716-aac4716. https://doi.org/10.1126/science.aac4716 Plonsky, L. (2012). Replication, meta-analysis, and generalizability. In G. K. Porte (Ed.), Replication Research in Applied Linguistics (pp. 116-132). Cambridge University Press. Porte, G. K. (Ed.). (2012). Replication research in applied linguistics. Cambridge University Press. 
Porte, G. K., \& McManus, K. (2019). Doing replication research in applied linguistics. Routledge.

Porte, G. K., \& Richards, K. (2012). Replication in second language writing research. Journal of Second Language Writing, 21(3), 284-293. https://doi.org/10.1016/j.jslw.2012.05.002

Saito, K., Macmillan, K., Mai, T., Suzukida, Y., Sun, H., Magne, V., Ilkan, M., \& Murakami, A. (2020). Developing, Analyzing and Sharing Multivariate Datasets: Individual Differences in L2 Learning Revisited. Annual Review of Applied Linguistics, 40, 9-25. https://doi.org/10.1017/S0267190520000045

Sanz, C., \& Morgan-Short, K. (2004). Positive Evidence Versus Explicit Rule Presentation and Explicit Negative Feedback: A Computer-Assisted Study: Language Learning. Language Learning, 54(1), 35-78. https://doi.org/10.1111/j.1467-9922.2004.00248.x

Schmidt, S. (2009). Shall we really do it again? The powerful concept of replication is neglected in the social sciences. Review of General Psychology, 13(2), 90-100. https://doi.org/10.1037/a0015108

SSLA. (2021, October 27). Open Science Badges. Studies in Second Language Acquisition. https://www.cambridge.org/core/journals/studies-in-second-language-acquisition/openscience-badges

Tracy-Ventura, N., McManus, K., Norris, J. M., \& Ortega, L. (2014). 'Repeat as much as you can': Elicited imitation as a measure of oral proficiency in L2 French. In P. Leclercq, H. Hilton, \& A. Edmonds (Eds.), Measuring L2 Proficiency: Perspectives from SLA (pp. 143-166). Multilingual Matters. 
Wu, S.-L., \& Ortega, L. (2013). Measuring global oral proficiency in SLA research: A new elicited imitation test of L2 Chinese. Foreign Language Annals, 46(4), 680-704. https://doi.org/10.1111/flan.12063

Yamada, Y. (2018). How to Crack Pre-registration: Toward Transparent and Open Science. Frontiers in Psychology, 9, 1831. https://doi.org/10.3389/fpsyg.2018.01831 